\title{
Aphid parasitoids (Hymenoptera, Braconidae, Aphidiinae) and their associations related to biological control in Brazil
}

\author{
Petr Starý ${ }^{1}$, Marcus Vinicius Sampaio ${ }^{2,3}$ \& Vanda Helena Paes Bueno ${ }^{2}$
}

\begin{abstract}
${ }^{1}$ Institute of Entomology, Czech Academy of Sciences, Branisovská 31, Ceské Budejovice 37005 Czech Republic. stary@entu.cas.cz ${ }^{2}$ Departamento de Entomologia, Universidade Federal de Lavras. Caixa Postal 37, 37200-000, Lavras-MG, Brazil. vhpbueno@ufla.br ${ }^{3}$ Present address: Instituto de Ciências Agrárias, Universidade Federal de Uberlândia, Campus Umuarama. Caixa Postal 593, 38400-902, Uberlândia-MG, Brazil. mvsampaio@iciag.ufu.br
\end{abstract}

\begin{abstract}
Aphid parasitoids (Hymenoptera, Braconidae, Aphidiinae) and their associations related to biological control in Brazil. This study evaluated the parasitoid-aphid-plant associations in Brazil with the objective of developing a useful research database for further studies of aphid parasitoid ecology and aphid management. The original material was obtained from collections made in Paraná, Rio Grande do Sul, Minas Gerais, and São Paulo states. The published information on the Aphidiinae in Brazil is revised. The general features of the target parasitoid fauna of Central and South America is summarized and promising biological control programs of some aphid species in Brazil is discussed.
\end{abstract}

KEYWORDS. Aphid; host range; insect-plant interactions.

\begin{abstract}
RESUMO. Parasitóides (Hymenoptera, Braconidae, Aphidiinae) de pulgões e suas interações relacionadas ao controle biológico no Brasil. Este trabalho avaliou as interações parasitóides-pulgão-planta no Brasil, com o objetivo de desenvolver um banco útil de dados para estudos subseqüentes em ecologia de parasitóides de afídeos e manejo de pulgões. O material original foi obtido de coletas nos estados do Paraná, Rio Grande do Sul, Minas Gerais e São Paulo, sendo revisadas as informações sobre Aphidiinae publicadas no Brasil. Foram também sumarizadas as caracteristicas gerais da fauna de parasitóides das Américas Central e do Sul e discutidos os programas potenciais de controle biológico de algumas espécies de pulgões no Brasil.
\end{abstract}

PALAVRAS-CHAVE. Afídeo; gama hospedeira; interação inseto-planta.

An overall research interest in Aphidiine parasitoids of aphids has increased all over the world. Aphid pest problems and ecological aspects of their control undoubtedly contributed positively to this state. Searches for potentially useful biological control agents in the pest origin center frequently have demonstrated relatively poor knowledge in many areas and the same has been determined in subsequent research efforts targeting parasitoid release and establishment. These studies have also demonstrated a need for broader ecological studies of the local fauna, ecological associations and inter-relationships, and also management problems. Aphidiine parasitoids were also found to be rather useful subjects for several fundamental studies. These studies may be essential for the applied research or stimulated by problems found in it.

Until the end of the 70's only three species of aphid parasitoids (Braconidae: Aphidiinae) Aphidius colemani Viereck, 1912, Diaeretiella rapae (M'Intosh, 1855), and Lysiphlebus testaceipes (Cresson, 1880) were known occur in Brazil (Araújo e Silva et al. 1968; Bartoszeck 1975, 1976a b; Pimenta \& Smith 1976; Gravena 1979; De Santis 1980; Lázzari 1985). The species on the genus Ephedrus Haliday were found parasitizing Brachycaudus persicae (Passerini) (Araújo e Silva et al. 1968). The parasitoid Aphidius platensis Brèthes, 1913 (Starý 1975) and other species mentioned in the South American literature (Aphidius chilensis Brèthes, 1912, Aphidius huebrichi Brèthes, 1913, Aphidius porteri Brèthes, 1915 and
Diaeretus porteri Brèthes, 1910) were recognized as synonyms of A. colemani (Starý 1995). Finally, Aphidius brasiliensis Brèthes, 1918 (Araújo e Silva et al. 1968, De Santis 1980) and Aphidius ohioensis Smith, 1944 (Bartoszeck 1975, 1976a) were, probably, a case of synonym and a misidentification of $A$. colemani, respectively (see below, review of parasitoid-aphidplant associations, to more details).

New introductions of aphid parasitoid species occurred in the end of the 70's within a framework of a biological control program of wheat aphids. Some biological control programs have yielded spectacular results in some South American countries and parasitoid contribution is also rather significant for aphid control in Brazil. Biological control of wheat aphids in Brazil is a good example of a successful program. It started in 1979 targeting the aphid species pests Metopolophium dirhodum (Walker, 1849), Schizaphis graminum (Rondani, 1852), and Sitobion avenae (Fabricius, 1775). Twelve aphidiine species were introduced in six States until 1992. The program was considered successful and high aphid populations on wheat crops in the 70's were drastically reduced in the 80's (Starý 1980; Gassen \& Tambasco 1983; Salvadori \& Salles 2002). Four species of aphid parasitoids were established in Rio Grande do Sul state (Aphidius ervi Haliday, 1834, Aphidius rhopalosiphi DeStefani-Perez, 1902, Aphidius uzbekistanicus Luzhetzki, 1960 and Praon volucre (Haliday, 1833)), but their host range was related mainly to grass aphids (Zuñiga-Salinas 1982). A more extensive study was made by Tavares (1991, 
1996) in São Paulo state, who recovered only P. volucre, from the introduced species, and three new records were added to aphid parasitoid species in Brazil (Aphidius rosae Haliday, 1834, Aphidius salicis Haliday, 1834, and Ephedrus persicae Froggatt, 1904).

After this period, very limited records focusing the postcolonization and the host range of aphid parasitoids on some cultivated plants were found in Brazil (Auad et al. 1997; SousaSilva et al., 1998; Lara et al. 1999; Fernandes et al. 2000; Mendes et al., 2000; Pinto et al. 2000; Cividanes 2002; Mussury \& Fernandes. 2002; Ronquim et al. 2004; Zanini et al. 2006), and still fewer were found on ornamentals (Peronti 1999; Imenes et al. 2002; Carvalho et al. 2006). Moreover, this information is scattered in the literature and a compilation of these data is necessary.

Cinara pinivora Wilson, 1919 and Cinara atlantica Wilson, 1919 were detected as pests on pine in Brazil in the end of 90's, but no parasitoids were found in the local fauna (Penteado et al. 2000). The parasitoid species Xenostigmus bifasciatus (Ashmead, 1891) was introduced from USA to the states of Paraná, Santa Catarina and São Paulo, in 2002 and 2003, and it has became established in Brazil, and dispersing $80 \mathrm{Km}$ per year from the release point (Reis-Filho et al. 2004; Oliveira 2006).

For these reasons, we have found useful to summarize the information about aphid-plant-parasitoid associations developing a useful research database for further studies. So, this study is focused in the associations of aphid parasitoids and their aphid and plant hosts in Brazil. For this, field collections of parasitized aphids were made in two southern states of Brazil (Paraná and Rio Grande do Sul) in 1980, during the wheat biological control program, and also in two southeastern states of the country (Minas Gerais and São Paulo) from 1999 to 2006, in an area where no releases of parasitoids were made during the biological programs of wheat and pine aphids. These field collections were made in cropping areas, greenhouses, avenues, parks and households, to obtain supplemental information of aphid-plant relationship for each parasitoid species. In order to complete the information on parasitoid-aphid-plant association in Brazil, literature data were revised, summarized, and doubtful records were explained based on the literature data, or derived from the experience of the authors. The general features of the parasitoid fauna of Central and South America were summarized and promising biological control programs of some aphid species in Brazil are discussed.

\section{MATERIALS AND METHODS}

Parasitoids were reared on identified host aphid-plant associations from field collections. Plant parts with aphid colonies were gently cut with scissors, placed in plastic containers covered with nylon mesh, and transferred to the laboratory. The emerging parasitoids were collected and preserved in ethanol $70 \%$. The sample data were arranged with the objective of developing a tri-trophic database. In each numbered sample, the information about location, date, aphid, plant and habitat was recorded.

Most of the material was obtained from the collections made within the framework of FAO biocontrol programs in Brazil (wheat aphids, Paraná, Rio Grande do Sul), and the others originated from studies by the authors, especially in Minas Gerais and São Paulo states.

Review of parasitoid-aphid-plant associations. The material is arranged according to host aphid names, subdivided by the States of Brazil. For our data from field collections the individual localities are listed alphabetically, presenting the location name, collection date, aphid host plant, habitat, collection number and the collector's name in brackets. Collector's names were abbreviated as follows: (MS) - M.V. Sampaio, (PS) - P. Starý, (VB) - V.H.P. Bueno. Other names are presented in full. Only the reference, in chronological order, and the host plant species were listed for data from the literature. At last, the whole published information on the target parasitoids was revised as much as possible based on up-dated knowledge. Incomplete information in literature resulting in doubtful parasitoid-aphid relationships are followed by a question mark (?). The opinions of the authors and comments about probable misidentifications are indicated by asterisk (*) after the species name. Aphid nomenclature followed Remaudiere \& Remaudiere (1997), in general.

Abbreviations of the Brazilian States are as follows: BA Bahia; MG - Minas Gerais; MS - Mato Grosso do Sul ${ }^{\dagger}$; PE Pernambuco; PR - Paraná †े $^{\circ}$ RJ - Rio de Janeiro; RS - Rio Grande do Sul ${ }^{\dagger}$; SC - Santa Catarina ${ }^{+\circ}$; SP - São Paulo ${ }^{{ }^{\circ}}$; Brazil - no State determined.

Only the States from where collections were made are listed. Parasitoids were released as part of wheat and pine aphids biocontrol programs in the States marked with dagger $\left(^{\dagger}\right)$ and a sun $\left({ }^{\circ}\right)$ sign, respectively. In the paragraph "Laboratory" results of laboratory observations on aphid-parasitoid relationships, which were not associated with faunal field data, are included.

\section{RESULTS}

\section{Review of parasitoid-aphid-plant associations}

Aphidius brasiliensis Brèthes, 1918*

*Probable synonym of Aphidius colemani Viereck - Even though we do not have access to the type material of this species and with only original description of Brèthes it is impossible to recognize this species, in our opinion, A. brasiliensis is a synonym of $A$. colemani. Our opinion is reinforced due to a general prevalence of A. colemani as a parasitoid species on A. fabae and B. schwartzi in the area.

Aphis fabae Scopoli, 1763

Brazil: Araújo e Silva et al. (1968), De Santis (1980).

Brachycaudus schwartzi (Börner, 1931)

PR: Bartoszeck (1976a), Prunus domestica, Prunus persica.

Macrosiphum euphorbiae (Thomas, 1878)

Brazil Araújo e Silva et al. (1968), De Santis (1980). 


\section{Aphidius colemani Viereck, 1912}

Aphis coreopsidis (Thomas, 1878)

SP: Tavares (1991), Bidens pilosa, Emilia sonchifolia.

Aphis craccivora Koch, 1854

MG: Lavras, 10-X-2001, Medicago sativa, field, TS/30 (MS). - Mendes et al. (2000), Medicago sativa.

Aphis fabae solanella Theobald, 1914

SP: Tavares (1991), Sessea brasiliensis, Solanum americanum. Tavares (1996), Solanum americanum, Sessea brasiliensis.

Aphis gossypii Glover, 1877

MG: Lavras, 28-X-2001, Theobroma cacao, greenhouse, TS/32 (MS). - Lavras, 7-VII-2002, Duranta repens aurea, city, TS/33 (MS). Lavras, 17-VII-2002, Gossypium hirsutum, field, TS/39 (MS). Lavras, 17-VII-2002, Hibiscus rosa-sinensis, city, TS/46 (MS).

RS: Passo Fundo, 22-IX-1980, Hibiscus sp., avenue, 80/53 (PS). Passo Fundo, 28-IX-1980, Hibiscus sp., avenue, 80/91 (PS).

SP: Santo Antônio de Posse, 18-VI-2001, Dendranthema grandiflora, greenhouse, TS/17 (A. Silva). - Tavares (1991), Ageratum conyzoides, Cyphomandra arabica, Schefflera sp., Tecomma sp. Tavares (1996), Hibiscus rosa-sinensis, Schefflera sp. - Peronti (1999), Dieffenbachia amoena, Hibiscus rosa-sinensis. - Imenes et al. (2002), Ixora macrothyrsa. - Carvalho et al. (2006), Dendranthema grandiflora?

Brazil: De Santis (1980).

Aphis hederae Kaltenbach, 1843

RS: Passo Fundo, 22-IX-1980, Hedera helix, garden - city, 80/48 (PS).

Aphis nerii Boyer de Fonscolombe, 1841

RS: Passo Fundo, 22-IX-1980, undetermined shrub, city, 80/47 (PS). Passo Fundo, 22-IX-1980, Nerium oleander, city park, 80/52 (PS). - Passo Fundo, 22-IX-1980, Nerium oleander, avenue, 80/54 (PS). - Passo Fundo, 22-IX-1980, Nerium oleander, garden, 80/55 (PS). - Santo Angelo, 18-IX-1980, Nerium oleander, park, 80/39 (PS).

SP: Tavares (1991), Asclepias curassavica, Nerium oleander. - Tavares (1996) Asclepias curassavica.

Brazil: De Santis (1980).

Aphis spiraecola Patch, 1914

MG: Juramento, 12-VIII-2002, Citrus sinensis, field, TS/49 (Rocha). Lavras, 21-VIII-2002, Citrus sinensis, orchard, TS/52 (MS).

SP: Tavares (1991), Baccharis dracunculifolia, Emilia sonchifolia, Erigeron bonariensis, Citrus sp., Rosa sp., Spirea sp., Tecomma sp.

Aphis sp.

MG: Lavras, 13-IX-2002, Callendula officinalis, garden, TS/57 (MS).

RS: Tapera, 23-IX-1980, Solanum sp., roadside, 80/72 (PS). - Passo Fundo, 1-X-1980, Spirea sp., park, 80/98 (PS). - Passo Fundo, 2X-1980, Pittosporum sp., park, 80/101 (PS). - Passo Fundo, 3-X1980, Buxus sp., park, 80/105 (PS). - Passo Fundo, 5-X-1980, Psidium sp., park, 80/108 (PS).

Brachycaudus helichrysi (Kaltenbach, 1843)

SP: Tavares (1991), Ageratum conyzoides, Baccharis dracunculifolia, Erechtites valerianaefolia, Erigeron bonariensis.

Brachycaudus schwartzi (Börner, 1931)

MG: Auad et al. (1997), Prunus persica.

PR: Bartoszeck (1976a), Prunus domestica, Prunus persica.

Brevicoryne brassicae (Linné, 1758)

SP: Tavares (1991), Lepidium virginicum.

Brazil: De Santis (1980).
Cavariella aegopodii (Scopoli, 1763)

SP: Tavares (1991), Foeniculum vulgare. - Tavares (1996), Foeniculum vulgare?

Diuraphis noxia (Mordvilko, 1913)*

Brazil: USDA material.

* Doubtful record - The occurrence of target aphid was not confirmed in Brazil.

Dysaphis apiifolia (Theobald, 1922)

SP: Tavares (1991), Petroselinum sativum.

Dysaphis tulipae (Boyer De Fonscolombe, 1841)

MG: Uberlândia, 26-VIII-2006, Belamcanda chinensis, city, TS/118 (Almeida).

Dysaphis sp.

SP: Carvalho et al. (2006), Dendranthema grandiflora?

Eucarazzia elegans (Ferrari, 1872)

MG: Lavras, 26-IX-2002, Salvia splendens, city, TS/65 (DeConti).

SP: Peronti (1999), Salvia splendens.

Hysteroneura setariae (Thomas, 1878)

MG: Uberlândia, 22-VII-2005, Oryza sativa, greenhouse, TS/113 (MS).

SP: Tavares (1991), Eleusine indica, Oryza sativa, Rhynchaelitrum roseum.

Macrosiphum euphorbiae (Thomas, 1878)

MG: Pinto et al. (2000), Solanum tuberosum?

Metopolophium dirhodum (Walker, 1849)

PR: Pimenta \& Smith (1976), Triticum sp. - Lázzari (1985) Hordeum sp.?

Myzus ornatus Laing, 1932

SP: Tavares (1991), Erechtites valerianaefolia, Erigeron bonariensis, Solanum americanum, Sonchus oleraceus. - Peronti (1999), Duranta repens aurea.

Myzus persicae (Sulzer, 1776)

MG: Ijací, 2-X-2002, Capsicum апnиum, greenhouse, TS/71 (Barbosa). - Lavras, 11-IX- 2000, Capsicum annuum, greenhouse, TS/7 (MS). - Lavras, 18-V-2001, Capsicum апnиum, greenhouse, TS/12 (MS). - Lavras, 24-IX-2001, Lactuca sativa, greenhouse, TS/23 (MS). Lavras, 20-VIII-2003, Duranta repens aurea, garden, TS/92 (DeConti). - São Gotardo, 22-VIII-2002, Solanum tuberosum, field, TS/51 (Leme). - Uberlândia, 13-VII-2006, Portulaca oleracea, city, TS/115 (MS) - Pinto et al. (2000), Solanum tuberosum?

SP: Santo Antônio de Posse, 23-VI-2001, Dendranthema grandiflora, greenhouse, TS/18 (A. Silva). - Tavares (1991), Ageratum conyzoides, Bidens pilosa, Brassica oleracea, Capsicum sp., Emilia sonchifolia, Erechtites valerianaefolia, Erigeron bonariensis, Lepidium virginicum, Solanum americanum. - Carvalho et al. (2006), Dendranthema grandiflora?

Nasonovia ribisnigri (Mosley, 1841)

MG: Três Pontas, 12-IX-2002, Lactuca sativa, field, TS/60 (Figueira).

Picturaphis vignaphila Blanchard, 1922

SP: Tavares (1991), Desmodium sp.

Rhopalosiphum maidis (Fitch, 1856)

PR: Lázzari (1985), Hordeum sp.

SP: Tavares (1991), Eleusine indica, Oryza sativa. - Ronquim et al. (2004), Avena sativa?

Brazil: De Santis (1980). 
Rhopalosiphum padi (Linné, 1758)

MG: Lavras, 13-VII-2003, Avena sativa, field, TS/88 (MS). - Lavras, 31-VII-2003, Avena sativa, field, TS/89 (MS).

PR: Lázzari (1985), Hordeum sp.

RS: Ernestina, 23-IX-1980, Triticum aestivum, field, 80/59 (PS). Marau, 29-IX-1980, Triticum aestivum, field, 80/94 (PS). - Passo Fundo, 23-IX-1980, Avena sativa, field, 80/77 (PS). - Passo Fundo, IX-1980, Triticum aestivum, EMBRAPA insectary glasshouse (PS). - Tio Hugo, 25-IX-1980, Triticum aestivum, field, 80/78 (PS). São Borja, 17-IX-1980, Triticum aestivum, field, 80/28 (PS).

SP: Ronquim et al. (2004), Avena sativa?

Schizaphis graminum (Rondani, 1852)

MG: Lavras, 24-VIII-1993, Sorghum sp. (VB). - Lavras, 2-X-2002, Triticum aestivum field, TS/68 (MS). - Lavras, 31-VII-2003, Avena sativa, field, TS/90 (MS).

PR: Lázzari (1985), Hordeum sp.

RS: Passo Fundo, 12-IX-1980, Triticum aestivum, EMBRAPA insectary glasshouse, 80/19 (PS). - São Borja, 16-IX-1980, Triticum aestivum, field, 80/21 (PS).

SP: Gravena (1979), Sorghum bicolor. - Ronquim et al. (2004), Avena sativa?

Brazil: De Santis (1980).

Sitobion avenae (Fabricius, 1775)

MG: Lavras, 2-X-2002, Triticum aestivum, field, TS/67 (MS).

PR: Pimenta \& Smith (1976), Triticum sp. - Lázzari (1985), Hordeum sp.?

Toxoptera aurantii (Boyer de Fonscolombe, 1841)

MG: Santo Antônio do Amparo, 14-X-2001, Coffea arabica, field, TS/ 31 (Ecole)

SP: Tavares (1991), Myricaria sp., Citrus sp. - Peronti (1999), Camellia japonica, Schefflera arboricola.

Toxoptera citricidus (Kirkaldy, 1907)

MG: Lavras, Citrus sp. (F. Leclant).

SP: Tavares (1991), Citrus sp., Zanthoxylum rhoefolium. - Tavares (1996), Citrus sp.

Brazil: De Santis (1980).

\section{Toxoptera $\mathrm{sp}$}

RS: Marau, 22-IX-1980, Citrus sp., orchard, 80/45 (PS). - Passo Fundo, 22-IX-1980, Citrus sp., garden 80/56 (PS). - Santa Maria, 22-IX1980, Citrus sp., garden, 80/58 (PS). - Santo Angelo, 18-IX-1980, Citrus sp., orchard, 80/38 (PS). - Santo Goncalves, 27-IX-1980, Citrus sp., garden, 80/87 (PS). - Sao Borja, 16-IX-1980, Citrus sp., forest edge, 80/278 (PS).

Uroleucon cordobense (Blanchard, 1932)

SP: Tavares (1991), Erigeron bonariensis.

Uroleucon erigeronense (Thomas, 1878)

SP: Tavares (1991), Erigeron bonariensis.

Cereal aphids

RS: Passo Fundo, IX-1980, EMBRAPA insectary (PS).

Without host records

SC: Seara, Nova Teutônia, $27^{\circ} 11^{\prime} \mathrm{S}, 52^{\circ} 23^{\prime} \mathrm{W}, 300-350 \mathrm{~m}$ alt. a.s.1. VII-1971 (Plaumann).

Laboratory

Gamarra et al. (1997), Myzus persicae. - Gonçalves-Gervásio et al. (2001), Schizaphis graminum. - Sampaio et al. (2001ab), Aphis gossypii, Myzus persicae. - Sampaio et al. (2003b), Aphis gossypii. - Sampaio et al. (2004), Myzus persicae. - Sampaio et al. (2005b), Aphis gossypii. - Bueno et al. (2006), Aphis gossypii, Myzus persicae, Rhopalosiphum maidis, Schizaphis graminum. - Pierre et al. (2006), Aphis gossypii. - Sampaio et al. (2006), Aphis gossypii, Schizaphis graminum.
Aphidius ervi Haliday, 1834

Acyrthosiphon kondoi Shinji, 1938

MG: Lavras, 10-X-2001, Medicago sativa, field, TS/27 (MS). - Mendes et al. (2000), Medicago sativa.

Acyrthosiphon pisum (Harris, 1776)

MG: Lavras, 10-X-2001, Medicago sativa, field, TS/27 (MS). - SousaSilva et al. (1998), Medicago sativa. - Mendes et al. (2000), Medicago sativa.

Acyrthosiphon sp.

MG: Lavras, 1993, Medicago sativa (VB).

Aphis gossypii Glover, 1877

SP: Lara et al. (1999), Solanum tuberosum?

Macrosiphum euphorbiae (Thomas, 1878)

MG: Lavras, 11-IX-2000, Capsicum annuum, field, TS/8 (MS).

SP: Lara et al. (1999), Solanum tuberosum?

Metopolophium dirhodum (Walker, 1849)

RS: Tio Hugo, 25-IX-1980, Triticum aestivum, field, 80/78 (PS).

Myzus persicae (Sulzer, 1776)

MG: Lavras, 11-IX-2000, Capsicum annuum, field, TS/7 (MS).

SP: Lara et al. (1999), Solanum tuberosum ?

Rhodobium porosum (Sanderson, 1901)

MG: Lavras, 16-VII-2002, Rosa sp., garden, TS/37 (MS).

Sitobion avenae (Fabricius, 1775)

MG: Lavras, 17-VIII-2003, Triticum aestivum, field, TS/91 (MS). Lavras, 28-VIII-2003, Triticum aestivum, field, TS/97 (MS).

RS: Tio Hugo, 25-IX-1980, Triticum aestivum, field, 80/78 (PS).

Cereal aphids

Laboratory stock, introduced as "EPL-79-50".

\section{Aphidius matricariae Haliday, 1834*}

*Doubtful identification - The record of this target species is doubtful due to a general prevalence of $A$. colemani as a parasitoid species on $A$. gossypii in the area, and to uncertain identification at that time.

Aphis gossypii Glover, 1877

Brazil: Mendes (1959)

\section{Aphidius ohioensis Smith, 1944*}

* Doubtful identification - The target species was previously found only in the Neartic region, with different host records, which does not include Brachycaudus schwartzi. Probably a misidentification of A. colemani due the prevalence of this species in B. schwartzi.

Brachycaudus schwartzi (Börner, 1931)

PR: Bartoszeck (1976a), Prunus domestica, Prunus persica.

Brazil: De Santis (1980).

\section{Aphidius rhopalosiphi DeStefani-Perez, 1902}

Metopolophium dirhodum (Walker, 1849)

RS: Bento Goncalves, 27-IX-1980, Triticum aestivum, field, 80/94 
(PS). - Ernestina, 23-IX-1980, Triticum aestivum, field, 80/59 (PS). - Ibiruba, 25-IX-1980, Triticum aestivum, field, 80/80 (PS). - Marau, 22-IX-1980, Triticum aestivum, field, 80/44 (PS). - Marau, 29-IX-19890, Triticum aestivum, field, 80/94 (PS). - Passo Fundo, 22-IX-1980, Lolium sp., waste place - roadside, in a park, 80/50 (PS). - Passo Fundo, 23-IX-1980, Triticum aestivum, field, 80/71 (PS). - Passo Fundo, 24-IX-1980, Triticum aestivum, field, 80/76 (PS). - Passo Fundo, 24-IX-1980, Avena sativa, field, 80/77 (PS). - Passo Fundo, 2-X-1980, Triticum aestivum, field, 80/102 (PS). Tio Hugo, 25-IX-1980, Triticum aestivum, field, 80/78 (PS). Tapera, 23-IX-1980, Triticum aestivum, field, 80/70 (PS). - Tapera, 23-IX-1980, Triticum aestivum, field, 80/71 (PS). - Zuñiga (1982).

Sitobion avenae (Fabricius, 1775)

RS: Bento Gonçalves, 27-IX-1980, Triticum aestivum, garden undergrowth, 80/88 (PS). - Ernestina, 23-IX-1980, Triticum aestivum, field, 80/54 (PS). - Espumoso, 25-IX-1980, Triticum aestivum, Field, 80/79 (PS). - Ibiruba, 25-IX-1980, Triticum aestivum, field, 80/80 (PS). - Marau, 22-IX-1980, Triticum aestivum, field, 80/44 (PS). - Marau, 29-IX-1980, Triticum aestivum, field, 80/?? (PS). - Passo Fundo, 24-IX-1980, Triticum aestivum, field, 80/74 (PS). - Passo Fundo, 24-IX-1980, Triticum aestivum, field, 80/76 (PS). - Passo Fundo, 24-IX-1980, Avena sativa, field, 80/77 (PS). - Tio Hugo, 25-IX-1980, Triticum aestivum, field, 80/78 (PS). - Tapera, 23-IX-1980, Triticum aestivum, field, 80/70 (PS). - Tapera, 23-IX-1980, Triticum aestivum, field, 80/71 (PS). - Zuñiga (1982).

Cereal aphids

RS: Passo Fundo, IX-1980, EMBRAPA insectary, Triticum aestivum (PS). - Passo Fundo, 1978, Triticum aestivum (E. Zuñiga).

Laboratory stock introduced from England. - Introduced as Aphidius sp. 1213.

\section{Aphidius rosae Haliday, 1834}

Macrosiphum rosae (Linné, 1758)

SP: Tavares (1991), Rosa sp.

\section{Aphidius salicis Haliday, 1834}

Cavariella aegopodii (Scopoli, 1763)

SP: Tavares (1991), Foeniculum vulgare. - Tavares (1996).

Cavariella sp.

RS: Bento Gonçalves, 27-IX-1980, Petroselinum carvifolia, garden, 80/89 (PS).

\section{Aphidius smithi Subba Rao and Sharma, 1959}

Acyrthosiphon pisum (Harris, 1776)

RS: Feliz, 27-IX-12980, Medicago sativa, field, 80/86 (PS). - Ijuí, 29VIII-1980, Leguminosae, garden, 80/92 (PS). - Ijuí, 18-IX-1980, Medicago sativa, field, 80/43 (PS). - Passo Fundo, IX-1980, EMBRAPA insectary, Vicia sp. (PS).

\section{Aphidius uzbekistanicus Luzhetzki, 1960}

Metopolophium dirhodum (Walker, 1849)

RS: Colorado, 25-IX-1980, Triticum aestivum, field, 80/85 (PS). Ernestina, 23-IX-1980, Triticum aestivum, field, 80/59, (PS). Ibiruba, 25-IX-1980, Triticum aestivum, field, 80/80 (PS). - Ijuí, 18-IX-1980, Triticum aestivum, field, 80/40 (PS). - Marau, 22IX-1980, Triticum aestivum, field, 80/44 (PS). - Passo Fundo, 24IX-1980, Triticum aestivum, field, 80/74 (PS). - Passo Fundo, 25IX- 1980, Triticum aestivum, Field, 80/78 (PS). - Passo Fundo, 19-
IX-1980, Triticum aestivum, field (Werner). - Tio Hugo, 25-IX1980, Triticum aestivum, field, 80/78 (PS). - Tapera, 23-IX-1980, Triticum aestivum, field, 80/69 (PS). - Tapera, 23-IX-1980, Triticum aestivum, field, 80/70 (PS). - Tapera, 23-IX-1980, Triticum aestivum, field, 80/71 (PS). - Zuñiga (1982).

Schizaphis graminum (Rondani, 1852)

MS: Gomez \& Rumiatto (1989a)

Sitobion avenae (Fabricius, 1775)

MG: Lavras, 2-X-2002, Triticum aestivum, field, TS/69 (MS). - Lavras, 17-VIII-2003, Triticum aestivum, field, TS/94 (MS). - Lavras, 28VIII-2003, Triticum aestivum, field, TS/95 (MS).

RS: Colorado, 25-IX-1980, Triticum aestivum, field, 80/85 (PS). Ernestina, 23-IX-1980, Triticum aestivum, field, 80/59 (PS). Espumoso, 25-IX-1980, Triticum aestivum, field, 80/79 (PS). Ibiruba, 25-IX-1980, Triticum aestivum, field, 80/85 (PS). - Ijuí, 18-IX-19890, Triticum aestivum, field, 80/40 (PS). - Marau, 22IX-1980, Triticum aestivum, field, 80/44 (PS). - Passo Fundo, 24IX-1980, Triticum aestivum, field, 80/74 (PS). - Passo Fundo, 24IX-1980, Triticum aestivum, field, 80/76 (PS). - Passo Fundo, 19IX-1980, Triticum aestivum, field (Werner). - Tio Hugo, 25-IX1980, Triticum aestivum, field, 80/78 (PS). - Tapera, 23-IX-1980, Triticum aestivum, field, 80/69 (PS). - Tapera, 23-IX-1980, Triticum aestivum, field, 80/70 (PS). - Tapera, 23-IX-1980, Triticum aestivum, field, 80/71 (PS). - Zuñiga (1982).

Cereal aphids

Laboratory stock introduced as EPL 79-66.

Laboratory

Gomez \& Rumiatto (1989b), Schizaphis graminum.

\section{Aphidius sp.}

SP: Moreti et al. (1984), Triticum aestivum.

\section{Binodoxys brevicornis (Haliday, 1883)}

Cavariella aegopodii (Scopoli, 1763)

MG: Lavras, 13-IX-2002, Foeniculum vulgare, garden, TS/54 (MS). Lavras, 18-IX-2002, Foeniculum vulgare, garden, TS/59 (DeConti). - Uberlândia, 04-IX-2005, Foeniculum vulgare, field, TS/112 (MS). Sampaio et al. (2003a), Foeniculum vulgare.

SP: Mococa, 20-XII-2004, Foeniculum vulgare, city, TS/110 (MS).

Hyadaphis foeniculi (Passerini, 1860)

MG: Lavras, 27-IX-2002, Apium leptophyllum, city, TS/64 (MS).

SP: Mococa, 14-XI-2005, Petroselinum crispum, city, TS/111 (MS).

\section{Binodoxys tucumanus (Starý, 1987)*}

* Identification needs confirmation - The target species was previously found only on Myzus persicae in Argentina. It is very close to another Neotropical species of Binodoxys which parasitizes Uroleucon aphids.

Uroleucon cordobense (Blanchard, 1932)

SP: Tavares (1991), Erigeron bonariensis.

Uroleucon erigeronense (Thomas, 1878)

SP: Tavares (1991), Erigeron bonariensis.

\section{Binodoxys sp.}

Uroleucon $\mathrm{sp}$.

RS: Passo Fundo, 24-IX-1980, Taraxacum sp., waste place, 80/75 (PS). 


\section{Diaeretiella rapae (M'Intosh, 1855)}

Acyrthosiphon kondoi Shinji, 1938

RS: Ijuí, 18-IX-19080, Medicago sativa, field, 80/43 (PS).

Acyrthosiphon pisum (Harris, 1776)

RS: Ijuí, 18-IX-1980, Medicago sativa, field, 80/43 (PS).

Aphis gossypii Glover, 1877

SP: Lara et al. (1999), Solanum tuberosum ?

Brevicoryne brassicae (Linné, 1758)

MG: Cana Verde, 14-IX-2002, Raphanus raphanistrum, field, TS/93 (Auad). - Juramento, 12-IX-2002, Brassica oleracea acephala, field, TS/66 (Rocha). - Lavras, 17-VI-2001, Raphanus raphanistrum, field, TS/14 (Mendes). - Lavras, 15-VII-2002, Brassica oleracea acephala, field, TS/36 (MS). - Lavras, 1-X2003, Nasturtium officinale, hidroponic greenhouse, TS/99 (MS). - Souza \& Bueno (1992), Brassica oleracea acephala. - Bueno \& Souza (1993), Brassica oleracea acephala. - Cividanes (2002), Brassica oleracea acephala.

MS: Mussury \& Fernandes (2002), Brassica napus.

RJ: Valença, 19-II-2000, Brassica oleracea acephala, city, TS/9 (MS).

SP: Santo Antônio de Posse, 30-IV- 2001, Brassica oleracea acephala, field, TS/11 (Carvalho). - Tavares (1991), Brassica oleracea, Lepidium virginicum.

Brazil: De Santis (1980).

Lipaphis erysimi (Kaltenbach, 1834)

MG: Cana Verde, 14-IX-2002, Raphanus raphanistrum, field, TS/93 (Auad). Lavras, 15-VII-2002, Brassica oleracea acephala, field, TS/41 (MS). - Lavras, 17-VI-2001, Raphanus raphanistrum, field, TS/14 (Mendes). - Lavras, 1-X-2003, Nasturtium officinale, hidroponic greenhouse, TS/100 (MS).

MS: Mussury \& Fernandes (2002), Brassica napus.

RJ: Seropédica, 26-VII-2002, Brassica oleracea acephala, field, TS/ 47 (MS).

RS: Passo Fundo, 3-X-1980, Brassica sp., field, 80/107 (PS).

Macrosiphum euphorbiae (Thomas, 1878)

MG: Pinto et al. (2000), Solanum tuberosum ?

SP: Lara et al. (1999), Solanum tuberosum?

Brazil: De Santis (1980).

Metopolophium dirhodum (Walker, 1849)

PR: Alves et al. (2005), Triticum aestivum ?. - Zanini et al. (2006), Triticum aestivum?

Myzus persicae (Sulzer, 1776)

MG: Cana Verde, 14-IX-2002, Raphanus raphanistrum, field, TS/93 (Auad). - Ijací, 2-X-2002, Capsicum annuum, greenhouse, TS/70 (Barbosa). - Lavras, 17-VII-2001, Raphanus raphanistrum, field, TS/14 (Mendes). - Lavras, 15-VII-2002, Brassica oleracea acephala, field, TS/35 (MS).- São Gotardo, 22-VIII-2002, Solanum tuberosum, field, TS/50 (Leme). - Lara et al. (1999), Solanum tuberosum?

RJ: Valença, 19-II-2000, Brassica oleracea acephala, city, TS/9 (MS).

SP: Santo Antônio de Posse, 21-V-2001, Dendranthema grandiflora, greenhouse, TS/16 (A. Silva). - Tavares (1991), Brassica oleracea, Lepidium virginicum, Solanum americanum, Sonchus oleraceus.

Rhopalosiphum maidis (Fitch, 1856)

PR: Alves et al. (2005), Triticum aestivum ?. - Zanini et al. (2006), Triticum aestivum?

SP: Ronquim et al. (2004), Avena sativa ?

Rhopalosiphum padi (Linné, 1758)

PR: Alves et al. (2005), Triticum aestivum ?. - Zanini et al. (2006), Triticum aestivum?
RS: São Borja, 16-IX-1980, Triticum aestivum, field, 80/25 (PS). SP: Ronquim et al. (2004), Avena sativa?

Sitobion avenae (Fabricius, 1775)

PR: Alves et al. (2005), Triticum aestivum ?. - Zanini et al. (2006), Triticum aestivum?

Schizaphis graminum (Rondani, 1852)

MS: Gomez \& Rumiatto (1989a).

SP: Gravena (1979), Sorghum bicolor. - Ronquim et al. (2004), Avena sativa?

Cereal aphids

Introduced laboratory stock EPL 79-65.

Laboratory

Bueno \& Souza (1992), Brevicoryne brassicae. - Pereira \& Lomônaco (2003), Brevicoryne brassicae.

\section{Ephedrus persicae Froggatt, 1904}

Aphis fabae solanella Theobald, 1914

SP: Tavares (1991), Solanum americanum.

Brachycaudus helichrysi (Kaltenbach, 1843)

SP: Tavares (1991), Erechtites valerianaefolia?

Myzus ornatus Laing, 1932

SP: Tavares (1991), Erechtites valerianaefolia?

Myzus persicae (Sulzer, 1776)

SP: Tavares (1991), Erechtites valerianaefolia?

Without host records

SP: Tavares (1996).

\section{Ephedrus plagiator (Nees, 1811)}

Acyrthosiphon pisum (Harris, 1776)

RS: Passo Fundo, IX-1980, EMBRAPA insectary, Vicia sp. (PS).

Metopolophium dirhodum (Walker, 1849)

RS: Passo Fundo, 19-IX-1980, Triticum aestivum, field (Werner). Passo Fundo, 30-IX-1980, Hordeum vulgare, field (de Salles).

Sitobion avenae (Fabricius, 1775)

RS: Passo Fundo, 19-IX-1980, Triticum aestivum, field (PS). - Passo Fundo, 30-IX-1980, Hordeum vulgare, field (de Salles). - Passo Fundo, 19-IX-1980, T. aestivum (Werner). São Borja, 16-IX-1980, Triticum aestivum, field (PS).

Cereal aphids

RS: Passo Fundo, IX-1980, Triticum aestivum, EMBRAPA insectary (PS). - Passo Fundo, 1978, Triticum aestivum (E. Zuñiga).

Laboratory stock- introduced as Ephedrus "novo".

Laboratory

Gomez \& Rumiatto (1989b), Schizaphis graminum.

Lysiphlebus testaceipes (Cresson, 1880)

Aphis coreopsidis (Thomas, 1878)

MG: Lavras, 26-XII-2002, Bidens pilosa, field, TS/74 (MS). - Lavras, 9-I-2003, Bidens pilosa, city, TS/80 (MS). - Lavras, 19-IV-2003, Bidens pilosa, city, TS/86 (MS)

SP: Tavares (1991), Bidens pilosa. 
Aphis craccivora Koch, 1854

MG: Lavras, 10-X-2002, Medicago sativa, field, TS/29 (MS).

Aphis fabae Scopoli, 1763

SP: Peronti (1999), Anthurium froebelii.

Aphis fabae solanella Theobald, 1914

MG: Uberlândia, 22-VIII-2006, Solanum americanum, city, TS/119 (MS).

SP: Tavares (1991), Sessea brasiliensis.

Aphis gossypii Glover, 1877

BA: Soares et al. (1999).

MG: Belo Oriente, 30-IV-2001, Eucalyptus sp., greenhouse, TS/19 (Zanetti). - Lavras, 7-VII-2002, Duranta repens aurea, city, TS/ 34 (MS). - Lavras, 15-VII-2002, Dendranthema grandiflora, greenhouse, TS/43 (MS). - Lavras, 15-VII-2002, Gossypium hirsutum, greenhouse, TS/42 (MS). - Lavras, 17-VII-2002, Gossypium hirsutum, field, TS/40 (MS). - Lavras, 17-VII-2002, Hibiscus rosa-sinensis, city, TS/45 (MS). - Lavras, 8-VIII-2002, Cucumis sativus, field, TS/48 (MS). - Lavras, 23-VIII-2002, Solanum tuberosum, field, TS/53 (MS). - Lavras, 18-IX-2002, Ageratum conyzoides, city, TS/61 (Figueira). - Lavras, 23-XII2002, Tibouchina granulosa, city, TS/76 (MS). - Lavras, 26-XII2002, Bidens pilosa, field, TS/75 (MS). - Lavras, 9-I-2003, Bidens pilosa, city, TS/79 (MS). - Lavras, 21-I-2003, Tibouchina granulosa, city, TS/82 (MS). - Nova Porteirinha, 13-V-2000, Gossypium hirsutum, field, TS/5 (Gonçalves). - Pitangui, 15-IX2003, Malpighia glabra, field, TS/98 (Moreno). - São Gonçalo do Sapucaí, 22-XI-2002, Abelmoschus esculentus, field, TS/77 (Figueira).

PE: Fernandes et al. (2000), Gossypium hirsutum.

RJ: Valença, 2-I-2003, Hibiscus rosa-sinensis, city, TS/78 (MS). Valença, 5-I-2003, Kalanchö̈ blossfeldiana, city, TS/84 (MS).

SP: Santo Antônio de Posse, 26-IV-2001, Dendranthema grandiflora, greenhouse, TS/20 (A. Silva). - Santo Antônio de Posse, 30-IV2001, Dendranthema grandiflora, greenhouse, TS/10 (A. Silva). - Santo Antônio de Posse, 7-V-2001, Dendranthema grandiflora, greenhouse, TS/21 (A. Silva). - Santo Antônio de Posse, 21-V2001, Dendranthema grandiflora, greenhouse, TS/15 (A. Silva). - São José do Rio Pardo, 23-XII-1999, Abelmoschus esculentus, field, TS/2 (MS). - São José do Rio Pardo, 03-X-2006, Malpighia glabra, city, TS/117 (MS).

- Tavares (1991), Cassia leptocarpa, Schefflera sp., Tecomma sp., Sessea brasiliensis. - Peronti (1999), Calliandra inaequilatera. Imenes et al. (2002), Ixora macrothyrsa. - Carvalho et al. (2006), Dendranthema grandiflora?

Brazil: De Santis (1980).

Aphis hederae (Kaltenbach, 1843)

SP: Peronti (1999), Schefflera arboricola.

Aphis nerii Boyer de Fonscolombe, 1841

MG: Lavras, 20-II-2003, Asclepias curassavica, waste place, TS/85 (DeConti).

SP: São José do Rio Pardo, 22-XII-1999, Nerium oleander, city, TS/1 (MS). - Tavares (1991), Nerium oleander. - Peronti (1999), Nerium oleander.

Brazil: De Santis (1980).

Aphis spiraecola Patch, 1914

MG: Barbacena, 21-IX-2002, Schefflera sp., city, TS/63 (Auad).

PR: Bartoszeck (1975), Pyrus malus.

SP: Tavares (1991), Tecomma sp. - Peronti (1999), Polyscia guilfoylei.

Aphis sp.

MG: Lavras, 19-IX-2002, Foeniculum vulgare, garden, TS/62 (MS).

Brevicoryne brassicae (Linné, 1758)

Brazil: De Santis (1980).
Dysaphis apiifolia (Theobald, 1922)

MG: Bambuí, 15-VI-2004, Petroselinum crispum, city, TS/120 (Figueira).

Dysaphis sp.

SP: Carvalho et al. (2006), Dendranthema grandiflora?

Hysteroneura setariae (Thomas, 1878)

MG: Uberlândia, 22-VII-2005, Oryza sativa, greenhouse, TS/114 (MS).

Longiunguis sacchari (Zehntner, 1897)

Brazil: De Santis (1980).

Metopolophium dirhodum (Walker, 1849)

PR: Alves et al. (2005), Triticum aestivum ?. - Zanini et al. (2006), Triticum aestivum.

Myzus persicae (Sulzer, 1776)

MG: Lavras, 31-VIII-1995 (D.Gamarra). - Lavras, 21-V-2001, Capsicum annuum, greenhouse, TS/13 (MS) - Lavras, 15-VII2002, Capsicum annuum, greenhouse, TS/44 (MS).

SP: Tavares (1991), Bidens pilosa, Emilia sonchifolia. - Carvalho et al. (2006), Dendranthema grandiflora?

Rhopalosiphum maidis (Fitch, 1856)

MG: Lavras, 4-X-2002, Sorghum bicolor, greenhouse, TS/73 (MS). Pitangui, 5-X-2003, Zea mays, field, TS/101 (Moreno).

PR: Alves et al. (2005), Triticum aestivum ?. - Zanini et al. (2006), Triticum aestivum.

SP: Ronquim et al. (2004), Avena sativa ?

Brazil: De Santis (1980).

Rhopalosiphum padi (Linné, 1758)

MG: Lavras, 31-VII-2003, Avena sativa, field, TS/89 (MS).

PR: Alves et al. (2005), Triticum aestivum ?. - Zanini et al. (2006), Triticum aestivum.

SP: Ronquim et al. (2004), Avena sativa?

Schizaphis graminum (Rondani, 1852)

MG: Lavras, 24-VIII-1993, Sorghum sp. (VB). - Lavras, 4-X-2002, Sorghum bicolor, greenhouse, TS/72 (MS). - Lavras, 31-VII-2003, Avena sativa, field, TS/90 (MS).

SP: Ronquim et al. (2004), Avena sativa ?

Brazil: De Santis (1980).

Sitobion avenae (Fabricius, 1775)

PR: Alves et al. (2005), Triticum aestivum ?. - Zanini et al. (2006), Triticum aestivum.

Toxoptera aurantii (Boyer de Fonscolombe, 1841)

SP: Tavares (1991), Chrysophyllum sp.

Toxoptera citricidus (Kirkaldy, 1907)

MG: Lavras, Citrus sp. (F. Leclant). -Lavras, 22-IX-2002, Citrus sp., city, TS/83 (Auad).

PR: Bartoszeck (1976b), Citrus sp.

SP: Mococa, 10-XII-2002, Citrus sinensis, city, TS/81 (MS).

Brazil: De Santis (1980).

Without host records

SP: Tavares (1996).

Laboratory

Araújo \& Moraes (1998), Aphis gossypii. - Rodrigues \& Bueno (2001), Aphis gossypii, Schizaphis graminum. - Rodrigues et al. (2001), Aphis gossypii, Schizaphis graminum. - Bueno et al. (2003a), Aphis gossypii, Myzus persicae. - Bueno et al. (2003b), Aphis gossypii. - Carnevale et al. (2003), Aphis gossypii, Myzus persicae. - Rodrigues et al. (2003ab), Schizaphis graminum. - Rodrigues et al. (2005), Aphis gossypii, Schizaphis graminum. - Bueno et al. 
(2006), Myzus persicae, Rhopalosiphum maidis, Schizaphis graminum. - Sampaio et al. (2006), Aphis gossypii, Schizaphis graminum

Praon gallicum Starý, 1971

Acyrthosiphon pisum (Harris, 1776)

RS: Passo Fundo, IX-1980, Vicia sp., EMBRAPA insectary (PS).

Schizaphis graminum (Rondani, 1852)

MS: Gomez \& Rumiatto (1989a).

Cereal aphids

RS: Passo Fundo, IX-1980, Triticum aestivum, EMBRAPA insectary (PS). - Passo Fundo, 1978, Triticum aestivum (E. Zuñiga).

Laboratory stock introduced as "Praon 68".

Introduced as EPL 79-76.

Laboratory

Gomez \& Rumiatto (1989b), Schizaphis graminum.

Praon volucre (Haliday, 1833)

Acyrthosiphon kondoi Shinji, 1938

MG: Lavras, 10-X-2001, Medicago sativa, field, TS/28 (MS).

RS: Ijuí, 18-IX-1980, Medicago sativa, field, 80/41 (PS). - Ijuí, 18IX-1980, Medicago sativa, field, 80/43 (PS).

Acyrthosiphon pisum (Harris, 1776)

MG: Lavras, 10-X-2001, Medicago sativa, field, TS/28 (MS).

RS: Ijuí, 18-IX-1980, Medicago sativa, field, 80/43 (PS).

Acyrthosiphon sp.

RS: Feliz, 27-IX-1980, Medicago sativa, field, 80/86 (PS). - Passo Fundo, 3-X-1980, Lupinus sp., field, 80/106 (PS).

Aulacorthum solani (Kaltenbach, 1843)

MG: Lavras, 24-IX-2001, Lactuca sativa, greenhouse, TS/25 (MS).

Brachycaudus helichrysi (Kaltenbach, 1843)

SP: Tavares (1991), Erechtites valerianaefolia?

Brevicoryne brassicae (Linné, 1758)

RS: Passo Fundo, 30-IX-1980, Brassica sp., field, 80/95 (PS).

Hyperomyzus lactucae (Linné, 1758)

SP: Tavares (1991), Sonchus oleraceus.

Hyperomyzus sp.

RS: Passo Fundo, 2-X-1980, Sonchus sp., park, 80/99 (PS).

Macrosiphum euphorbiae (Thomas, 1878)

MG: Andradas, 17-XI-2000, Rosa sp., greenhouse, TS/4 (VB). - Lavras, 11-IX-2000, Capsicum annuum, field, TS/6 (MS). - Lavras, 24IX-2001, Lactuca sativa, greenhouse, TS/25 (MS). - Lavras, 1-X2003, Lactuca sativa, hidroponic greenhouse, TS/102 (MS).

SP: Holambra, 10-VIII-2001, Rosa sp., greenhouse, TS/22 (VB).

Macrosiphum rosae (Linné, 1758)

MG: Lavras, 13-IX-2002, Rosa sp., garden, TS/55 (MS).

RS: Ibiruba, 25-IX-1980, Rosa sp., garden, 80/81 (PS).

Metopolophium dirhodum (Walker, 1849)

RS: Bento Gonçalves, 27-IX-19890, Triticum aestivum, field, 80/88 (PS). - Colorado, 25-IX-1980, Triticum aestivum, field, 80/85 (PS). - Ibiruba, 25-IX-1980, Triticum aestivum, field, 80/80 (PS). Marau, 22-IX-1980, Triticum aestivum, field, 80/44 (PS). - Passo
Fundo, 24-IX-1980, Triticum aestivum, field, 80/74 (PS). - Passo Fundo, 24-IX-1980, Triticum aestivum, field, 80/76 (PS). - Passo Fundo, 24-IX-1980, Avena sativa, field, 80/77 (PS). - Zuñiga (1982).

Cryptomyzus ribis (Linné, 1758)

MG: Lavras, 18-IX-2002, Leonurus sibiricus, garden, TS/58 (MS).

Myzus ornatus Laing, 1932

SP: Tavares (1991), Erechtites valerianaefolia.

Myzus persicae (Sulzer, 1776)

MG: Lavras, 31-VIII-19945 (D.Gamarra). - Lavras, 11-IX-2000, Capsicum annuum, field, TS/6 (MS). - Lavras, 24-IX-2002, Lactuca sativa, greenhouse, TS/25 (MS). - Lavras, 13-IX-2002, Callendula officinalis, garden, TS/56 (MS).

SP: Tavares (1991), Erechtites valerianaefolia?

Rhodobium porosum (Sanderson, 1901)

MG: Andradas, 20-V-2003, Rosa sp., TS/87 (VB). - Lavras, 16-VII2002, Rosa sp., garden, TS/38 (MS). - Lavras, 28-V-2003, Rosa sp., garden, TS/108 (Cavalcanti). - Lavras, 19-VI-2003, Rosa sp., garden, TS/109 (DeConti).

Sitobion avenae (Fabricius, 1775)

MG: Lavras, 28-VIII-2003, Triticum aestivum, field, TS/97 (MS).

RS: Bento Gonçalves, 27-IX-1980, Triticum aestivum, field, 80/88 (PS). Colorado, 25-IX-1980, Triticum aestivum, field, 80/85 (PS). - Ibiruba, 25-IX-1980, Triticum aestivum, field, 80/80 (PS). Marau, 22-IX-1980, Triticum aestivum, field, 80/44 (PS). - Passo Fundo, 24-IX-1980, Avena sativa, field, 80/77 (PS). - Passo Fundo, 24-IX-1980, Triticum aestivum, field, 80/76 (PS). - Passo Fundo, 24-IX-1980, Triticum aestivum, field, 80/74 (PS). - Zuñiga (1982).

Uroleucon cordobense (Blanchard, 1932)

SP: Tavares (1991), Erigeron bonariensis.

Uroleucon sonchi (Linné, 1767)

SP: Tavares (1991), Sonchus oleraceus.

Uroleucon sp.

MG: Lavras, 24-IX-2001, Lactuca sativa, greenhouse, TS/24 (MS).

RS: Passo Fundo, 12-IX-1980, Sonchus sp., waste place, 80/18 (PS). Passo Fundo, 2-X-1980, Sonchus sp., park, 80/99 (PS).

Cereal aphids

RS: Passo Fundo, IX-1980, Triticum aestivum, EMBRAPA insectary (PS).

Laboratory

Gomez \& Rumiatto (1989b), Schizaphis graminum.

\section{Xenostigmus bifasciatus (Ashmead, 1891)}

Cinara atlantica Wilson, 1919

PR: Reis-Filho et al. (2004), Pinus spp.

SC: Reis-Filho et al. (2004), Pinus spp.

SP: Reis-Filho et al. (2004), Pinus spp.

Cinara sp.

MG: Lavras, 16-VIII-2005, Pinus sp., avenue, TS/121 (Santos). Uberlândia, 17-IX-2006, Pinus sp., park, TS/116 (MS).

\section{Laboratory}

Reis-Filho et al. (2004), Cinara atlantica. - Oliveira (2006), Cinara atlantica. 


\section{DISCUSSION}

Fauna and their relationships. To understand the position of Brazil, a few general features on the aphidiine parasitoid fauna of Central and South America are presented. The following paragraph is generalized and derived from a set of published papers (Mackauer \& Starý 1967; Starý 1972; Aguilar 1980; De Huiza \& Ortu 1980; Starý 1981; Botto \& Hernández 1982; Starý \& Vogel 1985; Monguí et al. 1986; Starý 1987; Starý \& Delfino 1987; Valdiviesco Jara 1987; Starý \& Cermeli 1989; Gärdenfors 1990; Botto et al. 1991; Starý 1993; Starý et al. 1993; Starý 1994; Vergara Ruiz \& Galeano 1994; Yokomi et al. 1994; Guevara \& Zuluaga 1995; Starý 1995; Ravelo \& Triana 1997; Berta et al. 1998) as well as from original information by the authors. However, all the classification is rather preliminary due to the limited information from extensive areas of the Continent.

Apparently, Central America manifests relatively rich and mixed fauna partially penetrating from the southern parts of North America, apart from native elements and penetrations from South America. Similar features can also be observed in the Antilles, although species composition is less numerous (Cuba).

In South America, the Andean mountain range apparently has many specific fauna. Indications of such peculiar parasitoid fauna in the northern parts of South America are obvious. One of the peculiar complexes represents the Nothofagus associated fauna, which is also distributed in the lowlands in the north-south direction in the continent. The other distinct complex (under observation) is the parasitoid guild on Uroleucon aphids. Another peculiar fauna was indicated to occur in the subantarctics.

However, most of the aphidiine fauna of the continent is characterized by a typical complex consisting of several species (A. colemani, D. rapae and L. testaceipes) which is distributed widely both in the Atlantic and the Pacific coastal areas, penetrating far into the interior to even high altitudes. These parasitoid species are associated with common aphid species on many different crops, weeds, vegetables and ornamental plants both in the field and urban agglomerations.

The latter complex has been enriched by several species which were introduced as biocontrol agents and became wellestablished and dispersed beyond the target areas into the lowlands and into high altitude (A. ervi, Aphidius smithi Sharma \& Subba Rao, 1959, A. rhopalosiphi, A. uzbekistanicus, Ephedrus plagiator (Nees, 1811) and P. volucre).

Also, research has determined further exotic species that were accidentally introduced and became naturalized faunal elements (Aphidius matricariae Haliday, 1834, A. salicis and Binodoxys brevicornis (Haliday, 1883)).

The classification of the known Brazilian Aphidiine fauna demonstrated features corresponding to the Atlantic coastal areas and inland-distribution, enriched by the exotic species introduced accidentally and/or purposely. The faunal composition of the individual parasitoid-aphid associations is affected naturally and deeply by the climate zoning with the increasing numbers of species from the southern of the tropical belt.

Research outlines. The Aphidiine parasitoids were collected in as many environments as their host species could be found, in field crops, in greenhouses, and in non-crop plants. A. smithi was recorded for the first time in Brazil. Prior to the wheat aphid biocontrol program, the known parasitoid species in Brazil (A. colemani, D. rapae, and L. testaceipes) were reported as associated with many aphid pests such as Aphis gossypii Glover, 1877, Brachycaudus schwartzi (Börner, 1931), Brevicoryne brassicae (Linné, 1758), Myzus persicae (Sulzer, 1776), Rhopalosiphum maidis (Fitch, 1856), R. padi (Linné, 1758), and S. graminum (Brèthes 1913, 1918; Moreira 1919; Costa Lima 1936; Mendes 1959; Araújo e Silva et al. 1968; Bartoszeck 1975, 1976a, 1976b; Pimenta \& Smith 1976; Gallo et al. 1978; Gravena 1979; De Santis 1980). However, a literature review demonstrates how inequal information has been obtained from the different states of Brazil.

Non-target aphid species as alternative hosts of introduced parasitoid biocontrol agents were considered to be only secondary within the program network centered the individual target pests of wheat and pine. Even today, the actual distribution of introduced parasitoid species has remained unknown in many areas of Brazil. At least three biocontrol agents used in wheat biocontrol program (A. ervi, A. uzbekistanicus, and P. volucre) and one in pine aphid biological control program (X. bifasciatus) were found in Minas Gerais state, at least $300 \mathrm{Km}$ and $1000 \mathrm{Km}$ away from the release sites, respectively. Other parasitoid species (A. rhopalosiphi, Ephedrus plagiator (Nees, 1811) and Praon gallicum Starý, 1971) introduced for the biological program of wheat aphids, were found in original release sites and their establishment needs to be confirmed in Brazil.

The prevailing ability of many parasitoid species to develop on several host species was observed to contribute to their establishment, and it also represents an important resource for biological control programs in the future. Several promising targets are presented:

Preliminary studies on biological control of A. gossypii and M. persicae were done in Brazil (Sampaio et al. 2001a, 2001b; Rodrigues \& Bueno 2001; Rodrigues et al. 2001; Bueno et al. 2003a; Carnevale et al. 2003; Sampaio et al. 2003b, 2004, 2005). Good results have been obtained on controlling $A$. gossypii on commercial chrysanthemums under greenhouse conditions (Bueno et al. 2003b; Rodrigues et al. 2005).

Introduced parasitoid species specialized in Macrosiphini aphids (i.e. A. ervi and P. volucre) might be used on biological control of some aphid species, such as Acyrthosiphon kondoi Shinji, 1938, Acyrthosiphon pisum (Harris, 1776), Aulacorthum solani (Kaltenbach, 1843), and Macrosiphum euphorbiae (Thomas, 1878).

Research on Therioaphis trifolii (Monell, 1882), an exotic alfalfa pest showed a lack of parasitoids in Brazil (Sousa-Silva et al. 1998; Mendes et al. 2000). However, successful biocontrol has been achieved in some other parts of the world. These programs might also be applied in Brazil. 
Also, Uroleucon ambrosiae (Thomas, 1878) has become a serious lettuce pest (Auad et al. 2002). According to our observations, the pest status of this aphid has increased with the increase of hydroponics system in lettuce crops grown in greenhouses in Brazil. The authors have collected large colonies of this pest aphid on hydroponics lettuce without parasitoids. There are some indications of the presence of some native parasitoid species in Brazil, which should be preferred over the possibility of exotic introductions.

In general, pest aphids are usually the key position due to economical reasons. However, broader environmental studies on, inter alia, ecological aspects of pest control programs need to be done, focusing the necessary and useful interaction between the so-called fundamental and applied research.

Acknowledgments. The authors (M. V. Sampaio and V. H. P. Bueno) are in debit for scholarships and financial support by CNPQ (Brasil) and with Dr Carlos R. Sousa-Silva of UFSCar, São Carlos (SP) and Dr Rebeca Peña-Martines of Escuela Nacional de Ciencias Biologicas del Mexico (México) for the identification of part of the aphid species. A part of the results was achieved within the framework of research activities in FAO programmers TCP /BRA/ 8908 for Brazil and TCP/ CHI/0153 for Chile (consultancies by P. Starý). The research (P. Starý) was also partially supported by Grant S5007102 (Grant Agency of the Academy of Sciences of the Czech Republic) and from the Entomology Institute Project Z5007907 (Acad. Sci. of the Czech Rep.).

\section{REFERENCES}

Aguilar, P. G. 1980. Apuntes sobre el control biológico y el control integrado de las plagas agrícolas en el Perú. Revista Peruana de Entomologia 23: 83-101.

Alves, L. F. A.; T. M. V. Prestes; A. Zanini; M. F. Dalmolin \& A. O. Menezes Júnior. 2005. Controle biológico natural de pulgões (Hemiptera: Aphididae) em lavoura de trigo por parasitóides (Hymenoptera, Aphidiinae), no município de Medianeira, PR, Brasil. Semina: Ciências Agrárias 26: 155-160.

Araujo, L. H. A. \& A. C. S. Moraes. 1998. Parasitismo do pulgão do algodoeiro por Lysiphlebus testaceipes Cresson (Hymenoptera: Aphidiidae) em casa de vegetação. Pesquisa em Andamento EMBRAPA Algodão 92, 3p

Araújo e Silva, A. G.; C. R. Gonçalves; D. M. Galvão; A. J. L. Gonçalves; J. Gomes; M. N. Silva \& L. Simoni. 1968. Quarto catálogo de insetos que vivem nas plantas do Brasil. Seus parasitos e predadores. Parte II, $3^{\circ}$ tomo. Rio de janeiro, Ministério da Agricultura, xxvi+622p.

Auad, A. M., V. H. P. Bueno; C. M. Kato \& D. C. Gamarra. 1997. Ocorrência e flutuação populacional de predadores e parasitóides de Brachycaudus (Appelia) schwartzi (Börner) (Homoptera: Aphididae) em pessegueiro, em Jacuí, MG. Anais da Sociedade Entomológica do Brasil 26: 257-263.

Auad, A. M.; S. Freitas \& L. B. Barbosa, L. R. 2002. Ocorrência de afídeos em alface (Lactuca sativa L.) em cultivo hidropônico. Neotropical Entomology 31: 335-339.

Bartoszeck, A. B. 1975. Afídeos de macieira (Pyrus malus L.), seus predadores e parasitas. Acta Biológica Paranaense 4: 33-74.

Bartoszeck, A. B. 1976a. Afídeos da ameixeira (Prunus domestica L.) e pessegueiro (Prunus persica Sto.), seus predadores e parasitas. Acta Biológica Paranaense 5: 69-90.

Bartoszeck, A. B. 1976b. Afídeos de laranjeira (Citrus sinensis Osb.) e mimoseira (Citrus reticulata B.) seus predadores e parasitas. Acta Biológica Paranaense 5: 15-48.

Berta, D.C.; K. V. Colomo \& N. E. Ovruski. 1998. Interrelaciones entre los áfidos colonizadores del tomate y sus himenópteros parasitoides en Tucumán (Argentina). Boletin de Sanidade Vegetal de las Plagas 28: 67-77.

Botto, E. N. \& M. C. Hernández. 1982. Parasitismo de los áfidos Metopolophium dirhodum (Walker) y Sitobion avenae (F.) por parasitóides del género Aphidius (Hym., Aphidiidae) en la República Argentina. IDIA 401/404: 17-19.

Botto, E. N.; N. C Monetti \& A. R. Saluso. 1991. Introduction, colonization and establishment of Lysiphlebus testaceipes (Hymenoptera: Aphidiidae), in Argentina. Entomophaga 36: $323-$ 324.

Brèthes, J. 1913. Himenópteros de la América meridional. Annales del Museo Nacional de Historia Natural de Buenos Aires 24: $35-165$.

Brèthes, J. 1918. Sobre algunos Himenópteros útiles del Sur de Brasil. Annales de la Sociedad Rural de Argentina 52: 7-8

Bueno, V. H. P. \& B. M. Souza. 1992. Aspectos etológicos e longevidade de Diaeretiella rapae M'Intosh, 1855 (Hymenoptera: Aphidiidae). Revista de Agricultura 67: 49-54.

Bueno, V. H. P. \& B. M. Souza. 1993. Ocorrência e diversidade de predadores e parasitóides em couve (Brassica oleracea var. acephala) em Lavras, MG, Brasil. Anais da Sociedade Entomológica do Brasil 22: 5-18.

Bueno, V. H. P.; A. B. Carnevale \& M. V. Sampaio. 2003a. Host preference of Lysiphlebus testaceipes (Cresson) (Hym.: Aphidiidae) for Myzus persicae (Sulzer) and Aphis gossypii Glover (Hem.: Aphididae), p. 17-20. In: A. O. Soares; M. A. Ventura; V. Garcia \& J.-L. Hemptinne (ed.). Proceedings of the 8th International Symposium on Ecology of Aphidophaga: biology, ecology and behaviour of aphidophogous insects. Arquipélago, Life and Marine Science, $112 \mathrm{p}$.

Bueno, V. H. P.; J. C. Van Lenteren; L. C. P. Silveira \& S. M. M. Rodriguez. 2003b. An overview of biological control in greenhouse chrysanthemums in Brazil. Bulletin IOBC/WPRS 26: 1-5.

Bueno, V. H. P.; M. V. Sampaio; J. C. van Lenteren; B. F. De Conti; R. J. Silva; S. M. M. Rodrigues \& A. B. Carnevale. 2006. Evaluation of two aphid parasitoids as candidates for biocontrol of aphid pests in protected cultivation in Brazil. Bulletin IOBC/WPRS 29: 175180.

Carnevale, A. B.; V. H. P. Bueno \& M. V. Sampaio. 2003. Parasitismo e desenvolvimento de Lysiphlebus testaceipes (Cresson) (Hymenoptera: Aphidiidae) em Aphis gossypii Glover e Myzus persicae (Sulzer) (Hemiptera: Aphididae). Neotropical Entomology 32: 293-297.

Carvalho, L. M.; V. H. P. Bueno \& S. M. Mendes. 2006. Ocorrência e flutuação populacional de tripes, pulgões e inimigos naturais crisântemo de corte em casa de vegetação. Bragantia 65: 139146.

Cividanes, F. J. 2002. Impacto de inimigos naturais e de fatores meteorológicos sobre uma população de Brevicoryne brassicae (L.) (Hemiptera: Aphididae) em couve. Neotropical Entomology 31: $249-255$

Costa Lima, A. M. 1936. Terceiro catálogo dos insetos que vivem nas plantas do Brasil. Rio de Janeiro, Ministério da Agricultura.

De Huiza, I. R. \& M. S. Ortu. 1980. Algunos Aphidiinae (Hymenoptera: Braconidae) parasitoides de áfidos (Homoptera: Aphididae) en el Perú. Revista Peruana de Entomologia 23: 129-132.

De Santis, L. 1980. Catálogo de los himenópteros brasileños de la Serie Parasítica, incluindo Bethyloidea. Curitiba, Universidade Federal do Paraná, 395p.

Fernandes, A. M. V.; A. M. I. Farias; C. A. De Faria \& M. T. Tavares. 2000. Ocorrência de Aphelinus gossypii Timberlake (Hymenoptera: Aphelinidae) parasitando Aphis gossypii Glover (Hemiptera: Aphididae) em algodão no Estado de Pernambuco. Anais da Sociedade Entomológica do Brasil 29: 831-834.

Gallo, E.; O. Nakano; S. S. Neto; R. P. L. Carvalho, G. C. De Batista, E. Berti-Filho; J. R. P. Parra; R. A. Zucchi \& S. B. Alves. 1978 Manual de entomologia agrícola. São Paulo, Ceres, 531p.

Gamarra, D. C.; V. H. P. Bueno \& A. M. Auad. 1997. Efecto de los tricomas glandulares de Solanum berthaultii en el parasitismo de Aphidius colemani (Hymenoptera: Aphidiidae) sobre Myzus 
persicae (Homoptera: Aphididae). Vedalia 4: 21-23.

Gärdenfors, U. 1990. Trioxys apterus sp.n. from Ecuador, a new wingless species of Aphidiinae (Hymenoptera, Braconidae). Entomologia Scandinavica 21: $67-69$.

Gassen, D. N. \& F. J. Tambasco. 1983. Manejo Integrado de pragas em trigo. Informe Agropecuário 9: 47-49.

Gassen, D. N. \& F. J. Tambasco. 1983. Controle biológico dos pulgões do trigo no Brasil. Informe Agropecuário 9: 49-51.

Gomez, S. A. \& M. Rumiatto. 1989a. Avaliação do controle biológico de Schizaphis graminum (Rondani, 1852) por parasitos alienígenas introduzidos. Reunião da Comissão Centro-Sul-brasileira de pesquisa do trigo. Resultados da pesquisa com trigo-1988. UEPAED - EMBRAPA Agropecuária Oeste 39: 163-164 (Documentos).

Gomez, S. A. \& M. Rumiatto. 1989b. Criação e liberação de inimigos naturais para o controle de afídeos. Reunião da Comissão Centro-Sul-brasileira de pesquisa do trigo. Resultados da pesquisa com trigo-1988. UEPAED - EMBRAPA Agropecuária Oeste 39: 165-166 (Documentos).

Gonçalves-Gervásio, R. C. R.; L. V. C. Santa-Cecília; V. L. Carvalho; C. M. Kato; L. V. Foureaux \& M. G. Campelo. 2001. Efeito da idade da fêmea de Aphidius colemani Viereck (Hymenoptera: Aphidiidade) no parasitismo de Schizaphis graminum (Rondani) (Homoptera: Aphididae). Revista Ceres 48: 277-283.

Gravena, S. 1979. Dinâmica populacional do pulgão verde Schizaphis graminum (Rondani, 1852) (Homoptera, Aphididae) e inimigos naturais associados ao sorgo granífero em Jaboticabal, SP, Brasil. Anais da Sociedade Entomológica do Brasil 8: 325-334.

Guevara, G. R. \& C. J. I. Zuluaga. 1995. Reconocimiento de himenópteros parasitóides de áfidos en cultivos de algodón, cítricos y algunas hortalizas de Valle de Cauca. Revista Colombiana de Entomología 21: 71-76.

Imenes, S. D. L.; E. C. Bergmann; A. L. B. G. Peronti; S. Ide \& J. E. R. Martins. 2002. Aphids (Hemiptera: Aphididae) and their parasitoids (Hymenoptera) in Ixora spp. (Rubiaceae) in the states of Bahia and São Paulo, Brazil. Formal records of interactions. Arquivos do Instituto Biológico de São Paulo 69: 55-64.

Lara, F. M.; E. A. Silva \& A. L. Boiça-Júnior. 1999. Resistência de genótipos de batata, Solanum spp., a afídeos (Homoptera: Aphididae) e incidência sobre parasitóides. Anais da Sociedade Entomológica do Brasil 28: 721-728.

Lázzari, S. N. 1985. Inimigos naturais dos afídeos (Homoptera: Aphididae) da cevada (Hordeum sp.) no Paraná. Anais da Sociedade Entomológica do Brasil 14: 5-15.

Mackauer, M. \& P. Starý 1967. Hym., Ichneumonoidea. World Aphidiidae. In: V. Delucchi \& G. Remaudiere (ed.). Index of entomophagous Insects. Paris, Le Francois, 195p.

Mendes, L. O. T. 1959. Sobre a ocorrência de alguns inimigos naturais de insetos. Anais da Academia Brasileira de Ciências 31: 577-586.

Mendes, S.; M. N. Cervino; V. H. P. Bueno \& A. M. Auad. 2000. Diversidade de pulgões e de seus parasitóides e predadores na cultura da alfafa. Pesquisa Agropecuária Brasileira 35: 1305-1310.

Monguí, B.; J. E. Luque \& J. Escobar. 1986. Biología de Aphidius colemani (Hymenoptera: Aphidiidae) parasitóide de Myzus persicae (Homoptera: Aphididae) en crisantemos de la Sabana de Bogotá. Revista Colombiana de Entomolomologia 12: 46-53.

Moreira, C. 1919. Les pucerons et, leur oef d'hiver (Hem., Aphididae). Bulletin de la Societé Entomologique de France 1919: 236238 .

Moreti, A. C. C.; Z. A. Ramiro; M. I. G. Junquetti; C. C. Cacao; M. A. Cacao \& R. Buzolin Filho. 1984. Incidência de pulgões (Homoptera - Aphididae) em culturas de trigo na região agrícola de Marilia, Estado de São Paulo. Biológica 50: 1-8.

Mussury, R. M. \& W. D. Fernandes. 2002. Occurrence of Diaeretiella rapae (Mc'Intosh, 1855) (Hymenoptera: Aphidiidae) parasitising Lipaphis erysimi (Kaltenbach, 1843) and Brevicoryne brassicae (L. 1758) (Homoptera: Aphididae) in Brassica napus in Mato Grasso do Sul. Brazilian Archives of Biology and Technology 45: $41-46$.
Oliveira, S. 2006. Fatores biológicos e comportamentais do parasitóide Xenostigmus bifasciatus Ashmead 1891 (Hymenoptera, Braconidae) visando a otimização de criação massal em laboratório e índice de parasitismo em casa-devegetação. Dissertation, Curitiba, UFPR, 78 p.

Penteado, S. R. C.; R. F. Trentini; E. T. Iede \& W. Reis Filho. 2000. Pulgões do Pinus: nova praga florestal. Anais do $1^{\circ}$ Simpósio do Cone Sul sobre manejo de pragas e doenças do Pinus. Série Técnica IPEF 13: 97-102.

Pereira, C. D. \& C. Lomônaco. 2003. Produção de formas aladas de Brevicoryne brassicae (L.) (Hemiptera: Aphididae) por indução do parasitóide Diaeretiella rapae (McIntosh) (Hymenoptera: Aphidiidae) e alguns aspectos comportamentais da interação destas duas espécies. Neotropical Entomology 32: 97-102.

Peronti, A. L. B. G. 1999. Afídeos e cocóideos em plantas ornamentais na regiao de São Carlos - SP, seus parasitóides, predadores e suas associações com formigas. Dissertation, São Carlos, UFSCAR, 82 p.

Pierre, L. S. R.; V. H. P. Bueno; M. V. Sampaio; J. C. van Lenteren; B. F. De Conti; M. P. F. Silva \& L. C. P. Silveira. 2006. Intraguild predation between Orius insidiosus (Say) and Aphidius colemani Viereck, and biological control of Aphis gossypii Glover. Bulletin IOBC/WPRS 29: 219-222.

Pimenta, H. R. \& J. G. Smith. 1976. Afídeos, seus danos e inimigos naturais em platações de trigo (Triticum sp.) no estado do Paraná. Curitiba, OCEPAR, 175p.

Pinto, R. M.; V. H. P. Bueno \& L. C. Santa-Cecilia. 2000. Flutuação populacional de afídeos (Hemiptera: Aphididae) associados a cultura da batata, Solanum tuberosum L., no plantio de inverno em Alfenas, Sul de Minas Gerais. Anais da Sociedade Entomológica do Brasil 29: 649-657.

Ravelo, H. G. \& J. L. F. Triana. 1997. Enemigos naturales de Toxoptera citricidus (Kirkaldy) en el Oriente Cubano. Revista del Centro Agrícola 24: 78-80.

Remaudiere, G. \& M. Remaudiere. 1997. Catalogue of the World's Aphididae. Homoptera Aphidoidea. Paris, I.N.R.A., 473p.

Reis-Filho, W.; Penteado, S. R. C. \& Iede, E. T. 2004. Controle biológico de pulgão-gigante-do-pinus, Cinara atlantica (Hemiptera: Aphididae), pelo parasitóide, Xenostigmus bifasciatus (Hymenoptera: Braconidae). Comunicado Técnico 122, Colombo, Embrapa Floresta, 3 p.

Rodriguez, S. M. M. \& V. H. P. Bueno. 2001. Parasitism rates of Lysiphlebus testaceipes (Cresson) (Hym.: Aphidiidae) on Schizaphis graminum (Rond.) and Aphis gossypii Glover (Hem.: Aphididae). Neotropical Entomology 30: 625-629.

Rodrigues, S. M. M.; V. H. P Bueno \& J. S. S. Bueno Filho. 2001. Desenvolvimento e avaliação do sistema de criação aberta no controle de Aphis gossypii Glover (Hem.: Aphididae) por Lysiphlebus testaceipes (Cresson) (Hym.: Aphididae) em casa-de-vegetação. Neotropical Entomology 30: 433-436.

Rodrigues, S. M. M.; V. H. P. Bueno \& M. V. Sampaio. 2003a. Armazenamento de múmias de Schizaphis graminum (Rondani) (Hemiptera: Aphididae) parasitadas por Lysiphlebus testaceipes (Cresson) (Hymenoptera: Aphidiidae) em baixa temperatura. Boletín de Sanidad Vegetal - Plagas 29: 367-374.

Rodrigues, S. M. M.; V. H. P. Bueno \& M. V. Sampaio. 2003b. Tabela de vida de fertilidade de Lysiphlebus testaceipes (Cresson, 1880) (Hymenoptera, Aphidiidae) em Schizaphis graminum (Rondani, 1852) (Hemiptera, Aphididae). Revista Brasileira de Entomologia 47: 637-642.

Rodrigues, S. M. M.; V. H. P. Bueno \& M. V. Sampaio. 2005. Efeito da liberação inoculativa sazonal de Lysiphlebus testaceipes (Hym.: Aphidiidae) na população de Aphis gossypii (Hem.: Aphididae) em cultivo de crisântemo em casa de vegetação comercial. Boletin de Sanidad Vegetal Plagas 31: 199-207.

Ronquim, J. C.; Pacheco, J. M. \& Ronquim, C. C. 2004. Occurrence and parasitism of aphids (Hemiptera: Aphididae) on cultivars of irrigated oat (Avena spp.) in São Carlos, Brazil. Brazilian Archives of Biology and Technology 47: 163-169.

Salvadori, J. R. \& L. A. B. Salles. 2002. Controle Biológico dos Pulgões 
do Trigo, p. 427-448. In: J. R. P. Parra; P. S. M. Botelho; B. S. Corrêa-Ferreira \& J. M. S. Bento (ed.). Controle biológico no Brasil. São Paulo, Manole, 635 p.

Sampaio, M. V.; V. H. P. Bueno \& J. C. van Lenteren. 2001a. Preferência de Aphidius colemani Viereck (Hymenoptera: Aphidiidae) por Myzus persicae (Sulzer) e Aphis gossypii Glover (Hemiptera: Aphididae). Neotropical Entomology 30: 655-660.

Sampaio, M. V.; V. H. P. Bueno \& R. Pérez-Maluf. 2001b. Parasitismo de Aphidius colemani Viereck (Hymenoptera: Aphidiidae) em diferentes densidades de Myzus persicae (Sulzer) (Hemiptera: Aphididae). Neotropical Entomology 30: 81-87.

Sampaio, M. V.; V. H. P. Bueno; M. C. M. Soglia \& S. M. M. Rodrigues. 2003a. Ocorrência de Binodoxys brevicornis (Haliday, 1883) (Hymenoptera, Braconidae, Aphidiinae) no Brasil. Revista Brasileira de Entomologia 47: 649-651.

Sampaio, M. V.; V. H. P. Bueno; S. M. M. Rodrigues \& M. C. M. Soglia. 2003b. Thermal requirements of three populations of Aphidius colemani Viereck (Hymenoptera: Aphidiidae). Bulletin IOBC/ WPRS 26: 85-88.

Sampaio, M. V.; V. H. P. Bueno; S. M. M. Rodrigues \& M. C. M. Soglia. 2004. The effect of competition among aphid hosts for food on development time of Aphidius colemani Viereck (Hymenoptera: Aphidiidae), p. 371-374. In: J. S. Simon; C. A. Dedryver; C. Rispe \& M. Hullé (ed.). Aphids in a New Millennium. Versailles, INRA Editions, $549 \mathrm{p}$.

Sampaio, M. V.; V. H. P. Bueno; S. M. M. Rodrigues \& M. C. M. Soglia. 2005. Resposta à temperatura de Aphidius colemani Viereck (Hymenoptera, Braconidae, Aphidiinae) originário de três regiões climáticas de Minas Gerais, Brasil. Revista Brasileira de Entomologia 49: 141-147.

Sampaio, M. V.; V. H. P. Bueno; M. C. M. Soglia; B. F. De Conti \& S. M. M. Rodrigues. 2006. Larval competition between Aphidius colemani and Lysiphlebus testaceipes after multiparasitism of the host Aphis gossypii. Bulletin of Insectology 59: 147-151.

Soares, J. J.; O. S. Carvalho; D. M. P. Azevedo \& F. C. Freire. 1999. Manejo Integrado de pragas do algodão no Cerrado baiano. Anais II Congresso Brasileiro de Algodão, Campina Grande, Brasil, p. $5-10$.

Souza, B. M. \& V. H. P. Bueno. 1992. Parasitóides e hiperparasitóides de múmias de Brevicoryne brassicae (Linnaeus, 1758) (HemipteraHomoptera: Aphididae). Revista de Agricultura 67: 55-62.

Sousa-Silva, C. R.; J. M. Pacheco; J. B. Rassini \& F. A. Ilharco. 1998. Afídeos da alfafa no Brasil (Homoptera, Aphidoidea). Revista Brasileira de Entomologia 41: 285-288.

Starý, P. 1972. New aphid parasites (Hymenoptera: Aphidiidae) from Cuba. Annales Zoologici 29: 317-322.

Starý, P. 1975. Aphidius colemani Viereck: its taxonomy, distribution and host hange (Hymenoptera, Aphidiidae). Acta Entomologica Bohemoslovaca 72: 156-163.

Starý, P. 1980. Integrated control of wheat aphids. Project TCP (BRA) 8908(M). Mission report. Consultancy period Sept. 10Oct. 1980. Passo Fundo, EMBRAPA and FAO, 39pp.

Starý, P. 1981. Aphid parasitoids (Hymenoptera, Aphidiidae) from
Cuba. Acta Entomologica Bohemoslovaca 78: 33-42.

Starý, P. 1987. Subject bibliography of aphid parasitoids (Hymenoptera: Aphididae) of the world 1758-1982. Monographs to Applied Entomology. Journal of Applied Entomology (Supplements) 25: $1-101$.

Starý, P. 1993. The fate of released parasitoids (Hymenoptera: Braconidae: Aphidiinae) for biological control of aphids in Chile. Bulletin of Entomological Research 83: 633-639.

Starý, P. 1994. Aphid parasitoid fauna (Hymenoptera, Aphidiidae) of the southern beech (Nothofagus) forest. Studies on Neotropical Fauna and Environment 29: 87-98.

Starý, P. 1995. The Aphidiidae of Chile (Hymenoptera, Ichneumonoidea, Aphidiidae). Deutsches Entomologisches Zeitschrift N.F. 42: 113-138.

Starý, P. \& M. Cermeli. 1989. Parasitóides (Hym., Aphidiidae) de áfidos en plantas cultivadas de Venezuela. Boletín de Entomología Venezolano N.S. 5: 77-80.

Starý, P. \& M. A. Delfino. 1987. Parasitoids (Hym., Aphidiidae) of aphids (Hom., Aphididae) in Tucumán, Argentina. Bolletino de Laboratório di Entomologia Agricola "Filippo Silvestri" 43: 41-50.

Starý, P.; M. Gerding; H. Norambuena \& G. Remaudiere. 1993. Environmental research on aphid parasitoid biocontrol agents in Chile (Hym., Aphidiidae Aphelinidae). Journal of Applied Entomology 115: 292-306.

Starý, P. \& M. Vogel. 1985. Aphid parasitoids from the Sub-Antarctic. Spixiana 9: 25-31.

Tavares, M. T. 1991. Estudo das interações "plantas/afídeos/ parasitóide e hiperparasitóide" em ambientes naturais e antrópicos. Dissertation, São Carlos, UFSCAR, 65p.

Tavares, M. T. 1996. Ocorrência de Dendrocerus carpenteri (Curtis) (Hymenoptera: Megaspilidae) no Brasil. Anais da Sociedade Entomológica do Brasil 25: 363-364.

Valdiviesco Jara, L. W. 1987. Control biológico del pulgón verde de la alfalfa. 6 Bolletino Técnico INIPA, La Molina, Estación Experimental Agropecuaria, $18 \mathrm{p}$.

Vergara Ruiz, R. \& P. E. O. Galeano. 1994. Interacciones poblacionales entre áfidos y sus enemigos naturals en algodonero, en dos zonas del Tolima. Revista Colombiana de Entomología 20: 15-22.

Yokomi, R. K.; Y. Q. Tang; L. Nong \& M. L. Kok-Yokomi. 1994 Potential migration of the threat of the brown citrus aphid, Toxoptera citricidus (Kirkaldy), by integrated pest management. Proceedings of the Florida State Horticultural Society 106: 81-85.

Zanini, A.; T. M. V. Prestes; M. F. Dalmolin; L. F. A. Alves; \& A. O. Menezes Júnior. 2006. Ocorrência de Lysiphlebus testaceipes (Cresson) (Hymenoptera, Aphidiinae) parasitando pulgões (Hemiptera: Aphididae), em trigo em Medianeira, PR. Neotropical Entomology 35: 275-276.

Zuñiga, E. 1982. Controle biológico de afídeos do trigo (Homoptera: Aphididae) por meio de parasitóides no Planalto Médio do Rio Grande do Sul. Thesis, Curitiba, UFPR, 319 p.

Received 13/06/2006; accepted 13/02/2007 